\title{
Hydrogen-Induced Ostwald Ripening at Room Temperature in a Pd Nanocluster Film
}

\author{
M. Di Vece, ${ }^{1}$ D. Grandjean, ${ }^{1}$ M. J. Van Bael, ${ }^{1}$ C. P. Romero, ${ }^{1}$ X. Wang, ${ }^{1}$ S. Decoster, ${ }^{2}$ A. Vantomme, ${ }^{2}$ and P. Lievens ${ }^{1}$ \\ ${ }^{1}$ Laboratorium voor Vaste-Stoffysica en Magnetisme \& INPAC-Institute for Nanoscale Physics and Chemistry, Katholieke Universiteit \\ Leuven, B-3001 Leuven, Belgium \\ ${ }^{2}$ Instituut voor Kern- en Stralingsfysica \& INPAC-Institute for Nanoscale Physics and Chemistry, Katholieke Universiteit Leuven, \\ B-3001 Leuven, Belgium
}

(Received 23 December 2007; published 13 June 2008)

\begin{abstract}
The structural and morphological changes occurring in an ensemble of vapor deposited palladium nanoclusters have been studied after several hydrogenation cycles with x-ray diffraction, extended x-rayabsorption fine structure spectroscopy, Rutherford backscattering spectrometry, and STM. Initial hydrogenation increased the cluster size, a result that is attributed to hydrogen-induced Ostwald ripening. This phenomenon originates from the higher mobility of palladium atoms resulting from the low sublimation energy of the palladium hydride as compared to that of the palladium metal. The universality of this phenomenon makes it important for the application of future nanostructured hydrogen storage materials.
\end{abstract}

DOI: 10.1103/PhysRevLett.100.236105

The pursuit of high capacity hydrogen storage materials started long ago and gained new momentum due to the fossil fuel related problems. The use of hydrogen could contribute to a solution [1]. The main aim is obtaining high hydrogen concentrations in a material that possesses suitable transport properties under ambient conditions. A promising way to alter favorably the properties is by reducing the size to the extent that surface and quantum effects begin to play a major role. The investigation of nanocluster metal hydrides may therefore reveal novel properties. Palladium is one of the most widely studied metals with respect to hydrogen absorption. The structural and electronic changes of bulk palladium upon hydrogenation are well documented [2,3]. In palladium nanoclusters, the hydrogen miscibility gap is reduced considerably as a result of the nanoscale dimensions [4,5]. A reversible structural transformation has also been shown in stabilized palladium clusters when the hydrogen content is varied [6]. Moreover, at elevated temperatures palladium nanoclusters change size due to Ostwald ripening [7]: the larger clusters capture mobile atoms at the expense of smaller clusters [8]. As hydrogen in a metal can decrease the strength of the host metal bonding, this raises the question of how an ensemble of palladium nanoclusters will interact during and after hydrogenation. A morphological or structural change of the nanocluster ensemble may affect the hydrogenation properties as compared to a single cluster.

In this Letter we investigated the effect of hydrogen on a palladium cluster assembled film with three different techniques: $\mathrm{x}$-ray diffraction (XRD), extended $\mathrm{x}$-ray absorption fine structure (EXAFS), and scanning tunneling microscopy (STM). These three complementary methods were used to determine the size changes of the palladium nanoclusters upon exposure to hydrogenation and oxygen. The grain size was derived from the width of the XRD intensity peaks, the coordination number was obtained from EXAFS, and the (lateral) diameter of the nanoclusters was measured directly by STM. The x-ray absorption
PACS numbers: 68.47.Jn, 61.05.cj, 61.05.cp, 81.07.Bc

experiment yielded additional parameters such as interatomic distances, degree of disorder, and electronic information through the shift of the absorption edge; these support the observation of size changes. The experimental results are explained by the influence of hydrogen on the sublimation energy.

A dual-target dual-laser vaporization source [9-12] was used to prepare the nanocluster films. The palladium clusters of $4 \mathrm{~nm}$ diameter with a $50 \%$ size dispersion, as measured with the mass spectrometer, were deposited on $5 \mathrm{~mm} \times 5 \mathrm{~mm} \mathrm{Si}$ wafers with a $200 \mathrm{~nm} \mathrm{SiO}$ top layer. Previous studies on similar films show that the clusters maintain their size upon landing $[13,14]$. The base pressure in the deposition chamber was $6 \times 10^{-9}$ mbar. For comparison, a molecular beam epitaxy (MBE) film with a nominal thickness of $50 \mathrm{~nm}$ was deposited on a $\mathrm{Si}$ wafer with a native oxide layer. Rutherford backscattering (RBS) measurements show that the cluster film has a thickness of about $16 \mathrm{~nm}$, corresponding to about four nanocluster monolayers. Hydrogenation of the samples was achieved by replacing the argon storage gas by hydrogen (1 bar). Hydrogen was removed by exposure of the film to the ambient in which oxygen reacts with hydrogen. This treatment and the experiments were performed at room temperature.

The size of nanoclusters can be estimated from the width of the peaks in an XRD diffraction pattern using the Debye-Scherrer [15] formula, revealing the coherent scattering distance along the scattering vector, which is a good estimate of the grain diameter. XRD patterns, as shown in Fig. 1, were recorded in a grazing incidence configuration with a PANalytical X'Pert PRO x-ray diffractometer equipped with an ultrafast $X^{\prime}$ 'Celerator detector using $\mathrm{Cu}-K_{\alpha}$ radiation. The relatively broad reflections can be indexed with the fcc palladium metal structure. The cell parameters of all nonhydrogenated palladium samples are $0.391 \mathrm{~nm}[16]$ corresponding to a Pd-Pd first neighbor bond distance of $0.2758 \mathrm{~nm}$, in agreement with the literature 


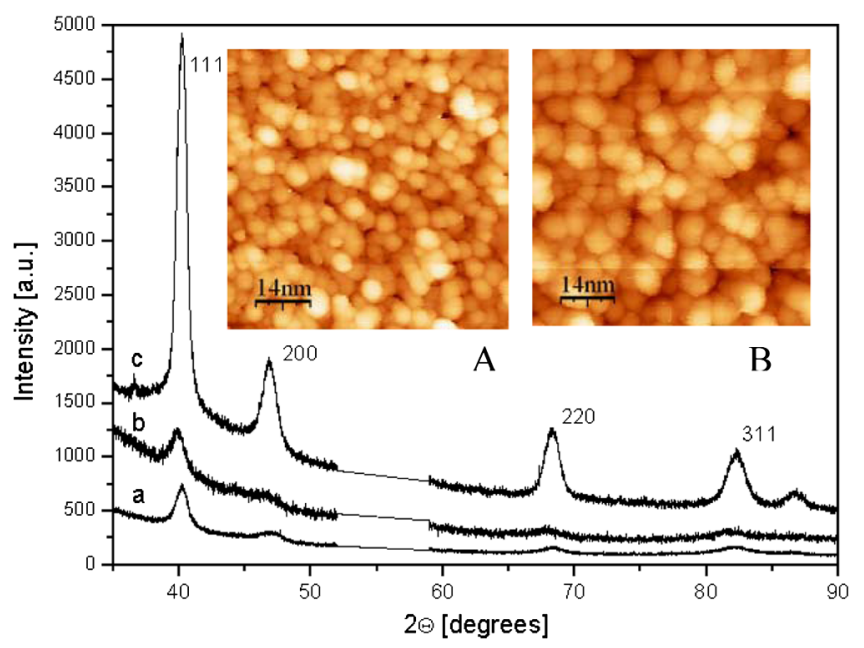

FIG. 1 (color online). XRD patterns of (a) an as-prepared cluster film, (b) the same film after $\mathrm{H}_{2}+\mathrm{O}_{2}$ treatment, (c) an as-prepared MBE film. The inset shows STM micrographs of palladium clusters (A) as-deposited on $\mathrm{SiO}_{2}$ and (B) after hydrogenation and oxygen exposure cycles.

range of $0.2751-0.2758 \mathrm{~nm}$ [17]. In the last column of Table I the diameter derived from the Debye-Scherrer formula is shown for each diffracting plane. In the cluster film the most intense (111) reflection reveals a grain size which is almost twice the size derived for the (200), (220), and (311) planes. This suggests that clusters do not remain strictly isolated but tend to form pairs or dimers connected through the fcc Pd (111) highest density plane. From the other diffraction peaks, grain sizes between 3.2 and $4.9 \mathrm{~nm}$ are obtained, in good agreement with the diameter of the clusters in the beam before deposition. After hydrogen and subsequent oxygen exposure, the Pd cluster size determined from the peak width (see Table I) has increased for all observed orientations, revealing an average increase of the cluster size of $22 \%$.

The average grain size in the MBE film as determined by $\mathrm{XRD}$, is $10.4 \mathrm{~nm}$. As in the nanocluster film, the (111) peak gives the largest size $(11.1 \mathrm{~nm})$. The other diffraction peaks reveal values between 6,8 and 9,3 $\mathrm{nm}$. Unfortunately, delamination of the MBE film after hydrogenation prevented measurements in the hydrogenated state.
EXAFS measurements provide a coordination number of the Pd atoms, which is determined by the surface-tovolume ratio $[18,19]$ and can be used to derive the size of the nanoclusters. $x$-ray absorption data were collected on the DUBBLE beam line (BM26A) at the European Synchrotron Radiation Facility (Grenoble, France) in fluorescence mode at the palladium $K$ edge $(24350.0 \mathrm{eV}$ with respect to vacuum) using a 9-channel Ge detector. Data reduction and analysis of the spectra were performed with the EXBROOK and EXCURV98 programs, respectively [20].

The first maximum of the palladium absorption $K$ edge in the XANES derivative shows a small shift towards lower energies $(1.6 \mathrm{eV})$ [21] for the as-prepared Pd cluster film $(24348.4 \mathrm{eV})$ compared to the $\mathrm{Pd}$ reference foil $(24350.0 \mathrm{eV})$; this has been observed before for palladium nanoclusters. Initial hydrogenation results in a further redshift by $1.6 \mathrm{eV}$, which can be attributed to the hydrogenpalladium bond shifting the band position as well as lowering the number of unoccupied $p$-like states near the Fermi level of Pd [22]. The as-prepared MBE-film absorption edge $(24350.0 \mathrm{eV})$, which is close to that of the reference Pd foil, also shifts significantly towards a lower energy $(4.7 \mathrm{eV})$. Subsequent hydrogenation and exposure to oxygen show no significant change, in agreement with the size stability, except for the second hydrogenation.

Phase-corrected Fourier transform of the $k^{3}$ weighted EXAFS spectra for both the cluster film and the MBE film together with the spectrum of the Pd reference foil are shown in Fig. 2. The main peak in these spectra corresponds to the Pd-Pd nearest-neighbor shell, which was only required to fit the EXAFS spectra. No other major peaks are present at higher $R$ values confirming the nanoscale character of these materials. The height of the peak is significantly reduced after the first hydrogenation, indicating a higher level of disorder in the metal structure. The intensity increases again after subsequent oxygen exposure but maintains its value after further hydrogen and oxygen exposure cycles. The hydrogenation treatments produce a marked shift of the main peak towards higher $R$ values; the Pd-Pd distance for the as-deposited cluster film increased from 0.2753 to $0.2820 \mathrm{~nm}$ upon hydrogenation, in good agreement with literature values at atmospheric pressure and room temperature [22]. Subsequent oxygen exposure

TABLE I. Analysis results of XRD measurements as shown in Fig. 1. for the cluster film as-prepared and after $\mathrm{H}_{2}$ and $\mathrm{O}_{2}$ exposures, and for the as-prepared MBE film. In addition to the average size, the sizes derived from the width (full width at half maximum) of the different diffraction peaks are given as well.

\begin{tabular}{|c|c|c|c|c|}
\hline Sample & Treatment & Cell parameter $(\mathrm{nm})$ & Pd-Pd bond distance $(\mathrm{nm})$ & Particle size (nm) \\
\hline \multirow[t]{2}{*}{ Clusters } & As-prepared & 0.3901 & 0.2758 & $\begin{array}{c}7.6(111), 4.1(200), 3.2(220), 4.9(311) \\
\text { Average size: } 4.7\end{array}$ \\
\hline & $\mathrm{H}_{2}+\mathrm{O}_{2}$ & 0.3924 & 0.2777 & $\begin{array}{c}9.3(111), 5.6(200), 4.2(220), 5.1(311) \\
\text { Average size: } 7.1\end{array}$ \\
\hline MBE-film & As-prepared & 0.3899 & 0.2757 & $\begin{array}{c}11.1(111), 8.2(200), 6.8(220), 9.3(311) \\
\text { Average size: } 10.4\end{array}$ \\
\hline
\end{tabular}



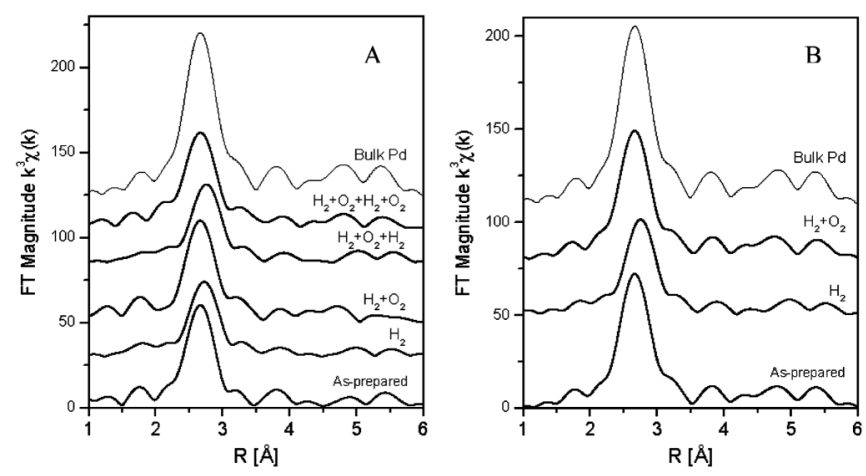

FIG. 2. Phase-corrected Fourier transforms of the $k^{3}$-weighted EXAFS as a function of the atomic distance $(R)$ of $(\mathrm{A})$ cluster (2.5 to $11 \AA^{-1}$ ) and (B) MBE-films (2.5 to $12 \AA^{-1}$ ) as-prepared and after various treatments. Amplitude reduction due to manyelectron processes (AFAC) was fixed at 0.85. The curves are vertically shifted for clarity.

and hydrogenation results in a reversible shortening (lengthening) of the Pd-Pd distance confirming complete loading (unloading), which otherwise would give a deviating nearest-neighbor distance.

The coordination number, 12 in a bulk fcc structure, was used to determine the size of the cluster. The Pd-Pd nearest-neighbor coordination number of 9.2 for the asdeposited nanocluster film, corresponds to a diameter of $4.7 \mathrm{~nm}$, in good agreement with the XRD and mass spectrometry results. A general trend of increased coordination number after the first hydrogenation treatment, converging to about $10(6.5 \mathrm{~nm})$ suggests an equilibrium state resulting from the hydrogenation and oxygen exposure. The enhanced coordination number after hydrogenation indicates a reduced surface-to-volume ratio and hence an increase of the cluster size of 38\%. The Debye-Waller factor that measures the structural disorder increases systematically after hydrogen exposure from $6.5 \times 10^{-5}$ to $8.5 \times 10^{-5} \mathrm{~nm}^{2}$ in the hydrogenated state in agreement with the literature for experiments at atmospheric pressure and room temperature [22].

The Pd-Pd distance for the as-deposited $(0.2754 \mathrm{~nm})$, hydrogenated $(0.2849 \mathrm{~nm})$, and oxidized $(0.2753 \mathrm{~nm}) \mathrm{MBE}$ film are in good agreement with literature values. The coordination number (and corresponding grain size) increases from $9.6(5.5 \mathrm{~nm})$ to $9.8(6.1 \mathrm{~nm})$ upon hydrogenation and decreases slightly upon oxygen exposure. As for to the nanocluster film, the level of structural disorder (Debye-Waller factor) also increases upon hydrogenation. The smaller grain size obtained from the EXAFS measurements $(5.5 \mathrm{~nm})$ compared to that from the XRD analysis $(10.4 \mathrm{~nm})$ for the as-prepared MBE film may be due to $\mathrm{X}$ ray self-absorption caused by the thicker Pd layer in the MBE film. This artificially lowers the intensity of the EXAFS oscillations, giving a smaller coordination number and smaller grain sizes. However, the comparison between the as-deposited and treated sample remains valid.
STM was used to determine directly the changes of the cluster diameter at the sample surface; in the micrographs (Fig. 1) the clusters are individually distinguishable, as was also observed by Binns, although many of them are in close contact with neighboring clusters in agreement with the larger cluster size derived from the XRD (111) diffraction peak. The clusters have a round, nearly spherical shape with an average diameter for the as-deposited cluster of $4.0 \mathrm{~nm}$, which is in good agreement with that measured with the mass spectrometer, indicating that this size is particularly stable. This was also observed in clusters formed by evaporation. The diameter of the nanoclusters after hydrogen treatment [Fig. 1(b)] has increased to $5.5 \mathrm{~nm}$ (increase of 37\%) corroborating the XRD and EXAFS results. The grains in the MBE film are less spherical and are more closely connected with an average size of about $8 \mathrm{~nm}$, in good agreement with the XRD values.

A clear increase of the nanocluster size was determined using three complementary techniques addressing the grain diameter along a specific direction (XRD), the cluster size via the coordination number and surface-to-volume ratio (EXAFS), and the lateral diameter of the clusters at the surface (STM). The increase of the nanocluster size measured with XRD, EXAFS, and STM is $22 \%, 38 \%$, and $37 \%$, respectively. This increase is much larger than the increase of about $8.1 \%$ in the Pd phase unit cell volume upon hydrogenation. Two possible mechanisms could lead to the increase of the nanocluster size. The small lattice expansion might increase the contact pressure, which could, by overcoming the surface tension, lead to a merging of the clusters. However, the STM micrographs clearly show that the clusters are packed randomly, with a space occupancy of only $64 \%$ [23]. Therefore, the nanoclusters have enough space to accommodate the small lattice expansion. Additionally, the film consists of about 4 nanocluster layers of which the top layers are considerably free to move and nevertheless show a size increase by STM.

A plausible mechanism for the cluster growth is the reorganization by three-dimensional Ostwald ripening, in which the larger clusters take up mobile atoms at the expense of smaller clusters. For most materials, spontaneous Ostwald ripening is an extremely slow process at room temperature. However, the presence of a hydrogen atom in the metal lattice reduces the binding energy, thus increasing the probability of detachment of palladium atoms. Since the clusters are in close contact, the detached atom does not have to travel far to stick to another cluster. These results therefore suggest that hydrogenation increases the mobility of palladium atoms. This process can be qualitatively understood in terms of the difference between the sublimation energy of palladium metal and palladium hydride. The sublimation energy of palladium decreases with increasing hydrogen concentration and is about $50 \%$ lower at a $\mathrm{H} / \mathrm{Pd}$ ratio of 0.3 [24]. In general, the sublimation rate $J$ of lattice atoms, at a temperature $T$, can be written as [25] 


$$
J=K_{0} n_{0} \exp \left(\frac{-E_{0}}{T}\right)
$$

where $n_{0}$ is the areal density of lattice atoms, $K_{0}=$ $k_{0} v_{\text {th }} / \lambda$, with $\lambda$ the lattice scale length, $v_{\text {th }}$ the thermal velocity of atoms, and $k_{0}$ a constant form factor of the order of unity and $E_{0}$ is the heat of sublimation. When the heat of sublimation is reduced by $50 \%$ by the hydrogenation of the palladium, the sublimation rate is increased by orders of magnitude. The effect of hydrogen on the palladium cluster sublimation rate is equivalent to a doubling of the temperature. Since our experiment was performed at room temperature a doubled temperature corresponds to approximately $600 \mathrm{~K}\left(327^{\circ} \mathrm{C}\right)$. At room temperature Ostwald ripening of palladium clusters is unlikely, while at $300{ }^{\circ} \mathrm{C}$ larger clusters are formed [26].

Although Ostwald ripening of palladium clusters upon hydrogenation has not been observed before due to protective layers or larger intercluster distances, there is some experimental evidence for the phenomenon. Inspection of published XRD spectra before and after hydrogenation of nanoclusters with diameters of 3.0 and $3.6 \mathrm{~nm}$ indicates a size increase to, respectively, 3.7 and $4.2 \mathrm{~nm}$ as derived from the Debye-Scherrer formula [27]. The strong morphological change in small palladium islands upon hydrogenation, reported by Xu et al. [28], could also be the result of hydrogen-induced Ostwald ripening.

The grains of the MBE film are much larger than those of the cluster film. The small variation in size during treatment cycles may be explained by the MBE process itself that involves the deposition of single palladium atoms. This leads to a thermodynamically stable configuration through diffusion and ripening. On the other hand the as-deposited clusters are in a metastable configuration, which changes by a lowering of the diffusion barrier on hydrogenation. Under changed conditions the ripening towards larger cluster size is therefore very likely. The relatively small grain size variation of the MBE film upon hydrogenation confirms the idea of a preferred grain size, which is in agreement with various studies of metal deposition on oxide surfaces [29].

In conclusion, we find that nanoclusters formed in the gas phase maintain their integrity upon soft landing, which indicates a metastable state for the cluster. Upon hydrogenation the average cluster size increases by $32 \%$. This increase is attributed to Ostwald ripening induced by exposure to hydrogen: the absorbed hydrogen decreases the sublimation energy of palladium, which stimulates the detachment of atoms from the clusters at room temperature. Hence, an individual nanocluster may have favorable hydrogen storage and transport properties but these may be lost as a result of morphological changes caused by hydrogenation cycles. Since this is very likely a general phenomenon, the exposure of nanostructured materials to hydrogen may need to be viewed from this perspective.
The authors acknowledge the assistance and advice of S. Nikitenko during the EXAFS measurements, and J. Fitzsche for providing the Pd MBE film. Access to the synchrotron facilities at the ESRF (BM26 DUBBLEexperiment 26-01-773) was supported by the Belgian Fund for Scientific Research-Flanders. The Flemish Concerted Action (No. GOA/2004/02), and the Federal Interuniversity Attraction Poles (No. IAP/V/1 and No. IAP/VI/42) Research Programs financed this work.

[1] L. Schlapbach and A. Züttel, Nature (London) 414, 353 (2001).

[2] J. Völkl and G. Alefeld, Hydrogen in Metals I, edited by G. Alefeld and J. Völkl, Topics in Applied Physics Vol. 28 (Springer, Berlin, 1978).

[3] E. Wilke and H. Brodowsky, Hydrogen in Metals II, edited by G. Alefeld and J. Völkl, Topics in Applied Physics Vol. 29 (Springer, Berlin, 1978).

[4] J. A. Eastman, L. J. Thompson, and B. J. Kestel, Phys. Rev. B 48, 84 (1993).

[5] A. Pundt et al., J. Alloys Compd. 293-295, 480 (1999).

[6] A. Pundt et al., Eur. Phys. J. D 19, 333 (2002).

[7] A. Howard, C. E. J. Mittchell, and R. G. Egdell, Surf. Sci. 515, L504 (2002).

[8] W. Ostwald, Z. Phys. Chem. (Leipzig) 34, 495 (1900).

[9] H. Hövel et al., Phys. Rev. B 48, 18178 (1993).

[10] C. Binns, Surf. Sci. Rep. 44, 1 (2001).

[11] W. Bouwen et al., Rev. Sci. Instrum. 71, 54 (2000).

[12] P. Milani and W. A. de Heer, Rev. Sci. Instrum. 61, 1835 (1990).

[13] C. Hendrich et al., Appl. Phys. A 86, 533 (2007).

[14] A. N. Dobrynin et al., Appl. Phys. Lett. 87, 012501 (2005).

[15] A. L. Patterson, Phys. Rev. 56, 978 (1939).

[16] M. Suleiman et al., J. Alloys Compd. 356-357, 644 (2003).

[17] Y. Sun et al., Langmuir 22, 807 (2006).

[18] A. I. Frenkel, C. W. Hills, and R. G. Nuzzo, J. Phys. Chem. B 105, 12689 (2001).

[19] R. E. Benfield, J. Chem. Soc., Faraday Trans. 88, 1107 (1992).

[20] N. Binsted et al., EXAFS Analysis Programs (Daresbury Laboratory, Warrington, U.K., 1991).

[21] See EPAPS Document No. E-PRLTAO-100-035825 for detailed information of the time of flight mass spectrum, x-ray absorption edge, EXAFS parameters, and STM images. For more information on EPAPS, see http:// www.aip.org/pubservs/epaps.html.

[22] R. J. Davis et al., Phys. Rev. B 39, 10580 (1989).

[23] W. N. Arnold, Science 288, 55 (2000).

[24] N. V. Piskunov et al., J. Eng. Phys. Thermophys. 74, 1217 (2001).

[25] R. P. Doerner, S. I. Krasheninnikov, and K. Schmid, J. Appl. Phys. 95, 4471 (2004).

[26] C. M. Whelan and C. J. Barnes, Appl. Surf. Sci. 119, 288 (1997).

[27] A. Pundt et al., Mater. Sci. Eng. B 108, 19 (2004).

[28] T. Xu et al., Appl. Phys. Lett. 86, 203104 (2005).

[29] C. T. Campbell, Surf. Sci. Rep. 27, 1 (1997). 